\title{
Ruxolitinib Regimen
}

National Cancer Institute

\section{Source}

National Cancer Institute. Ruxolitinib Regimen. NCI Thesaurus. Code C160116.

A chemotherapy regimen consisting of ruxolitinib that may be used in the treatment of myeloproliferative neoplasms. 